\title{
On Chilean Loxosceles (Araneae: Sicariidae): first description of the males of $L$. surca and $L$. coquimbo, new records of $L$. laeta and three remarkable new species from coastal deserts
}

\author{
Antonio D. BRESCOVIT ${ }^{1, *}$, Andrés TAUCARE-RÍOS ${ }^{2}$, \\ Ivan L. F. MAGALHAES ${ }^{3} \&$ Adalberto J. SANTOS ${ }^{4}$ \\ ${ }^{1}$ Laboratório Especial de Coleções Zoológicas, Instituto Butantan, São Paulo, Brazil. \\ ${ }^{2}$ Centro de Investigación en Medio Ambiente (CENIMA), Universidad Arturo Prat, \\ Casilla 121, Iquique, Chile. \\ ${ }^{3}$ División Aracnología, Museo Argentino de Ciencias Naturales "Bernardino Rivadavia", \\ Buenos Aires, Argentina. \\ ${ }^{4}$ Departamento de Zoologia, Instituto de Ciências Biológicas, \\ Universidade Federal de Minas Gerais, Minas Gerais, Brazil. \\ *Corresponding author: antonio.brescovit@butantan.gov.br \\ ${ }^{2}$ Email: and.taucare26@gmail.com \\ 32Email: magalhaes@macn.gov.ar \\ ${ }^{4}$ Email: oxyopes@yahoo.com

\footnotetext{
1 urn:lsid:zoobank.org:author:29156EF8-63CA-4499-8CB7-C57C20A9109B

${ }^{2}$ urn:lsid:zoobank.org:author:00F90C36-13E5-40E5-B0BB-F07D71DDC5D8

${ }^{3}$ urn:lsid:zoobank.org:author:799DF5B9-9085-4E58-BE36-371CFADED8AA

${ }^{4}$ urn:1sid:zoobank.org:author:531D2B5C-B6E6-46A4-ACBA-3DCD9FD0FDBF
}

\begin{abstract}
Loxosceles Heineken \& Lowe, 1832 spiders are infamous for their medical importance, but a taxonomic picture of the genus is still far from complete. In this study, the Chilean species of Loxosceles are described and mapped. The males of Loxosceles surca Gertsch, 1967 and L. coquimbo Gertsch, 1967 are described for the first time. Three new species with narrow distributions are described from central and northern Chile: Loxosceles diaguita sp. nov. from the Antofagasta Region, L. pallalla sp. nov. from Coquimbo and L. vallenar sp. nov. from Atacama. The first two species are remarkable in their morphology and do not fit into any of Gertsch's species groups, suggesting that Chile still harbours an undiscovered phylogenetic diversity of the genus. New distribution records for Loxosceles laeta (Nicolet, 1849) are provided throughout Chile.
\end{abstract}

Keywords. Brown recluse spiders, violin spiders, Haplogynae.

Brescovit A.D., Taucare-Ríos A., Magalhaes I.L.F. \& Santos A.J. 2017. On Chilean Loxosceles (Araneae: Sicariidae): first description of the males of $L$. surca and $L$. coquimbo, new records of $L$. laeta and three remarkable new species from coastal deserts. European Journal of Taxonomy 388: 1-20.

https://doi.org/10.5852/ejt.2017.388 


\section{Introduction}

The spider genus Loxosceles Heineken \& Lowe, 1832 is well-known for the dermonecrotic effects of their venom (Vetter 2008; Silva et al. 2004), figuring among the most frequent agents of arachnid envenomation in South America (Silva et al. 2015). The genus currently contains 116 species distributed mostly in the USA, Mexico, the Caribbean and South America. A few species have been described or reported from the African mainland, the Canary Islands and China, and at least two species are widespread and synanthropic (World Spider Catalog 2017). Considering its importance as an agent of envenomation and the biotechnological interest on its toxins (Senff-Ribeiro et al. 2008), one could imagine the genus is particularly well known taxonomically. However, the systematics of the genus is still problematic in most of its distribution range. This is particularly true regarding the South American fauna, which contains a third of the species of the genus (World Spider Catalog 2017).

The most recent revision of the South American Loxosceles was published 50 years ago (Gertsch 1967). Nevertheless, this study is still relevant for species recognition in the region due to the quality of its illustrations and detailed descriptions. However, most species included in Gertsch's revision remain poorly known regarding both its morphology and geographic distribution. This situation is well illustrated by the Chilean species of the genus. The first species described for the country, Loxosceles laeta (Nicolet, 1849) is well known for its current widespread distribution (Gertsch 1967; World Spider Catalog 2017), its association with domestic habitats (Schenone et al. 1970; Fischer et al. 2011; Taucare-Ríos et al. 2013) and, as a consequence, for being responsible for most dermonecrotic arachnid envenomation accidents in South America (Schenone et al. 1989; Schenone 2004). On the other hand, two other species recorded in the country are known only from a few taxonomic reports. Loxosceles coquimbo Gertsch, 1967 is known only from the type locality and has never been reported again in the literature after its original description (Gertsch 1967; World Spider Catalog 2017). Most recently, Loxosceles surca Gertsch, 1967, a species described from two nearby localities in Peru only, has been recorded from northern Chile (Taucare-Ríos 2011). Considering the well-recognized sampling deficiencies on South American spiders, it is open to speculation whether these species are truly short-range endemics or whether they have not been recorded of their entire distribution area. Additionally, both species are known so far only from the females, which might hamper their identification.

In this study, we contribute to the advancement of the knowledge on the Chilean Loxosceles by describing the males of $L$. coquimbo and $L$. surca. We also complement the known distribution of L. laeta by providing new records. Most remarkably, we describe three new short-ranged species, a demonstration that the small number of species of the genus in the country is a result of sampling deficiency and lack of taxonomic studies in this group.

\section{Material and methods}

\section{Methodology}

Terminology, format of descriptions and leg spination follow Sánchez-Ruiz \& Brescovit (2013). All measurements are in millimetres. Digital multifocus photos were taken using a Leica DFC 500 digital camera attached to a Leica MZ16A stereo microscope and a Leica M165C stereo microscope. The photographs were assembled using the software Leica Application Suite Version 3.3.0 and Helicon Focus 6 (available from http://www.heliconsoft.com/heliconsoft-products/helicon-focus/). SEM images were taken with a FEI Quanta 250 Scanning Electron Microscope at the Instituto Butantan. After dissection, female genitalia were placed in clove oil, examined and illustrated using a Leica MZ12 stereo microscope with a drawing tube. 
Geographic coordinates were obtained using a GPS for specimens collected by us, or through information on museum specimen original labels. Localities from museum samples without coordinates were georeferenced using Google Earth ${ }^{\circledR}$.

\title{
Institutional abbreviations
}

The material examined in this study is deposited in the following collections (curators in parentheses).

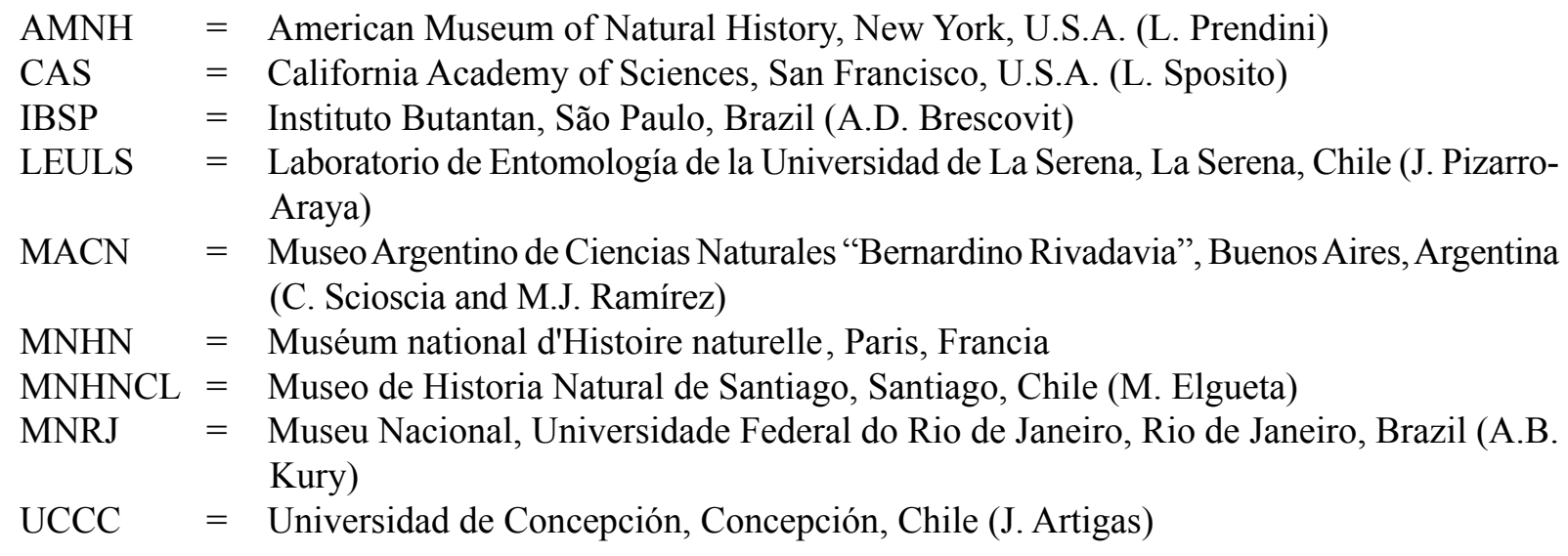

\section{Results}

\author{
Class Arachnida Lamarck, 1801 \\ Order Araneae Clerck, 1757 \\ Family Sicariidae Keyserling, 1880 \\ Genus Loxosceles Heineken \& Lowe, 1832
}

Loxosceles laeta (Nicolet, 1849)

Figs 1A, 9A, 10

Scytodes laeta Nicolet, 1849: 349 (female syntypes from Chile, supposedly deposited in MNHN, probably lost).

Loxosceles laeta - Simon 1900: 49. — Gertsch 1967: 147-152, pl. 6, figs 1-9, pl. 7, figs 1-5, 8-9 (full synonymy list). - World Spider Catalog 2017.

\section{Diagnosis}

Loxosceles laeta could be confused in Chile only with the sympatric L. surca. It is easily distinguished from $L$. surca by the anterior border of the carapace not too hirsute, the male palp with tibia medially incrassate and four times longer than the cymbium (Fig. 1A). The female internal genitalia are very similar to those of $L$. surca, but can be recognized by the transverse enlargement at the base of the tubular receptacle, which is usually angled or produced into a distinct tubercle or short blind, finger-like projection (see Gertsch 1967: pl. 6, figs 3-4, 6-9).

\section{Material examined}

CHILE. I Región de Tarapacá: Tamarugal, 1 q, Huara, Pisagua, 19.6 S, 70.21667 W, 15 Nov. 1941, J. Bird leg. (AMNH); Iquique, 2 우, Iquique city, 20.21389 S, 70.1525 W, May 2002, G. Arriagada leg.

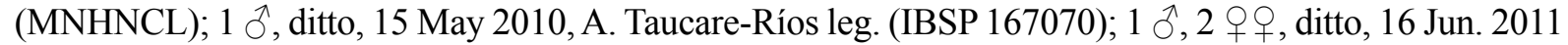
(IBSP 167072); 1 Oे, Altoda, Usmagama, 19.743056 S, 69.219722 W, 3400 m a.s.l., 9 Feb. 1993, L.E. Peña leg. (AMNH); 2 우, 1 immature (imm.), Punta Patache, 20.81067 S, 70.18725 W, 19 m a.s.1., 13 Jul. 2012, 
A.D. Brescovit, A.J. Santos and A. Taucare-Ríos leg. (IBSP 167064); 3 우, 20 imms, Pisagua, Punta

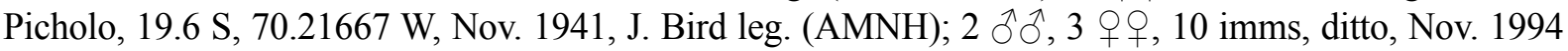
(AMNH); Tamarugal, 1 §̂, Huara, Quebrada Tarapacá, 19.92333 S, 69.50883 W, 1416 m a.s.1., 11 Jul. 2012, A.D. Brescovit, A.J. Santos and A. Taucare-Ríos leg. (IBSP 167065); 1 ô, 5 웅, 15 imms, Pozo Almonte, La Tirana, La Huayca, 20.43567 S, 69.54533 W, 990 m a.s.l., 10 Jul. 2012, A.D. Brescovit, A.J. Santos and A. Taucare-Ríos leg. (IBSP 167057); 1 O̊, 3 우, 9 imms, Pica, Canchones, Centro Experimental Canchones, near La Huayca, 20.445 S, 69.53383 W, 982 m a.s.1., 10 Jul. 2012, A.D. Brescovit, A.J. Santos and A. Taucare-Ríos leg. (IBSP 167062); 5 우, 9 imms, Tarapacá, Humberstone, 20.20581 S, 69.79406 W, 1983, E.A. Maury leg. (MACN 21477). - II Región de Antofagasta: Antofagasta 1 đa, 1 q, María Elena, 21.91667 S, 69.8 W, 1938 (AMNH); 7 đิ ô, 17 우, 6 imms, Antofagasta, 23.65 S, 70.4 W, 1989, J. Vidal leg. (IBSP 4663); 1 +, 1 imm., La Chimba, 23.52132 S, 70.37787 W, 268 m a.s.l., 15 Jul. 2012, A.D. Brescovit, A.J. Santos and A. Taucare-Ríos leg. (IBSP 167067); 1 ð, 2 우, 4 imms, Vertedero Municipal, 23.533 S, 70.3833 W, 100 m a.s.l., 15 Jul. 2012, A.D. Brescovit, A.J. Santos and A. Taucare-Ríos leg. (IBSP 167060); 1 ô, 8 imms, Mejillones, Mejillones, 23.105 S, 70.46267 W, 23 m a.s.l., in garbage, 14 Jul. 2012, A.D. Brescovit, A.J. Santos and A. Taucare-Ríos leg. (IBSP 167055); 2 đ̊̄, 3 우, 4 imms, Taltal, 25.283333 S 69.766667 W, 24 Oct. 1985, G. Arriagada leg. (MHNS); 3 qq , 1 imm., Taltal, 5 km from Taltal, 25.34698 S, 70.44578 W, 24 m a.s.1., in a cave by the road, 17 Jul. 2012, A.D. Brescovit, A.J. Santos and A. Taucare-Ríos leg. (IBSP 167056); 6 우, Caleta Hueso, 25.39083 S, 70.47361 W, 28 Jan.-4 Feb. 1941, J. Bird leg. (AMNH); 1 + 1 imm., Paposo, 22.9 S, 69.31667 W, 2002, O. León leg. (MNHNCL). - III Región de Atacama: Chañaral, 1 ㅇ, 3 imms, Chañaral, 26.35577 S, 70.63795 W, 12 m a.s.l., 17 Jul. 2012, A.D. Brescovit, A.J. Santos and A. Taucare-Ríos leg. (IBSP 167058); Copiapó, 1 q, 3 imms, Atacama, Copiapó, Caleta Pajonales, 27.73533 S, 70.98895 W, 141 m a.s.1., 10 Nov. 2013, A.D. Brescovit et al. leg. (IBSP 167079); 2 q 9 , Caldera, Calderilla, 27.0936 S, 70.85217 W, 25 m a.s.1., 10 Nov. 2013, A.D. Brescovit et al. leg. (IBSP 167087); 2 우, 4 imms, Puerto Viejo, 27.33708 S, 70.93197 W, 85 m a.s.l., 10 Nov. 2013, A.D. Brescovit et al. leg. (IBSP 167083); Huasco, 1 ðิ, 3 웅, 2 imms, Atacama, Caleta Chañaral de Aceituno, 29.0788 S, 71.48782 W, $18 \mathrm{~m}$ a.s.1., 11 Nov. 2013, A.D. Brescovit et al. leg. (IBSP 167075); 1 Oे, 3 imms, Carrizalillo, 29.10455 S, 71.40623 W, 124 m a.s.1., 11 Nov. 2013, A.D. Brescovit et al. leg. (IBSP 167081); 1 § , 3 우, 5 imms, Vallenar, $27 \mathrm{~km} \mathrm{~W}$ of Vallenar, $28.56667 \mathrm{~S}, 70.75 \mathrm{~W}, 105 \mathrm{~m}$ a.s.l., 7 Jan. 1985, N. Platnick and O. Francke leg. (AMNH); 2 우 imms, El Tránsito, 28.8769 S, 70.2805 W, 1100-1600 m a.s.l., 25-27 Oct. 1980, L.E. Peña leg. (AMNH); 1 ㅇ, 1 imm., Freirina, 28.5 S, 71.0667 W, 27-28 Oct. 1980, L.E. Peña leg. (AMNH). - IV Región de Coquimbo: Choapa, 1 ㅇ, Coquimbo Canela Baja, 31.40575 S, 71.46003 W, 7 Nov. 2013, A.D. Brescovit et al. leg. (IBSP 167080); 1 \&, Salamanca, La Jonquera, 31.76667 S, 70.96667 W, 19-20 Jul. 1961, D. Gonzalez leg. (AMNH); Coquimbo, 2 ㅊㅀ, 3 우우, 4 imms, Coquimbo, 29.95 S, 71.3334 W, Mar. 2002, H. Niemeyer leg. (MNHNCL); Elqui, Coquimbo, 1 ㄱ, 2 웅, 29.90778 S, $71.25417 \mathrm{~W}$, no date, R. Wagenknecht leg. (MNRJ 58027); 1 ㅅ, 6 우, 3 imms, La Higuera, Caleta Hornos, 29.62733 S, 71.28072 W, 8 Nov. 2013, A.D. Brescovit et al. leg. (IBSP 167082); 1 q, Chungungo, 29.4446 S, 71.30283 W, 34 m a.s.1., 11 Nov. 2013, A.D. Brescovit et al. leg. (IBSP 167085); 1 đ, Valle del Elqui, Los Choros, 29.23333 S, 71.45 W, 6-9 Sep. 2007, J. Pizarro-Arraya et al. leg. (LEULS); 1 ㅇ, La Serena, Compania Baja, Llanos, 29.91667 S, 70.96667 W, 16 Jun. 2007, A. Gonzales and Y. Laques leg. (LEULS); 1 q, ditto (LEULS); 1 +, 1 imm., La Serena, Colina El Pino, 29.9 S, 70.2432 W, 13 Apr. 2010, Grupo 1 Lab. leg. (LEULS); 1 क , ditto, 29.91135 S, 70.2432 W, 57 m a.s.l., 16 Aug. 2013, M. Hurtado leg. (LEULS); 1 o, ditto, 29.9 S, 71.25 W, 6 Aug. 1961, A.F. Archer leg. (AMNH); 1 ㅇ, La Serena, 29.9 S, 70.2432 W, 20 Jul. 2013, P.A. Róten leg. (LEULS); 1 đ, 1 ㅇ, 3 imms, Sector Parque Cori, 29.9 S, 71.23333 W, 12 May 2011, P. Yera B. leg. (LEULS); 1 , , Valle del Elqui, La Marqueza, 29.94158 S, $70.95792 \mathrm{~W}$, in a farm, 2008, J. Pizarro-Arraya et al. leg. (LEULS); 1 q, Vicuña,

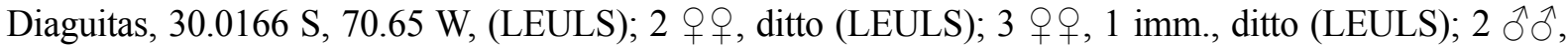

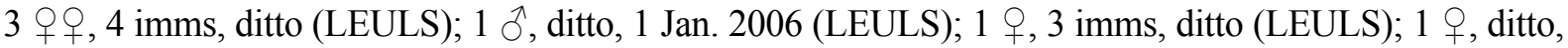
13 Aug. 2004 (LEULS); 1 ㅇ, ditto, 18 Sep. 2006 (LEULS); 1 ภ, 1 q, ditto, Jan. 2008 (LEULS); 1 o, 1 , ditto, 19 Sep. 2007, L.S.C. and J.V. leg. (LEULS); Limarí, 3 우, 5 imms, Combarbalá, La Ligua Baja, 31.0251 S, 71.04028 W, 7 Sep. 2013, A.D. Brescovit et al. leg. (IBSP 167076); 1 q, Ovalle, Camiño a Fray 
Jorge, 30.59622 S, 71.65365 W, 388 m a.s.1., 12 Nov. 2013, A.D. Brescovit and I.L.F. Magalhaes leg. (IBSP 167084); 1 q, 2 imms, Ovalle, 30.58333 S, 71.2 W, 6 Aug. 2005, P. Augusto leg. (LEULS); 1 q, 8 imms, ditto, 30.56783 S, 71.18175 W, 337 m a.s.1., 8 Nov. 2013, A.D. Brescovit et al. leg. (IBSP 167074). - RM, Región Metropolitana de Santiago: Santiago, 1 ㅇ, Santiago, Cerro Blanco, 33.41963 S, 70.63333 W, Oct. 1961, Franczinek leg. (AMNH); 1 q, El Canelo, Cajón del Maipo, 33.63333 S, 70.35 W, 850 m a.s.l., Nov. 1984, L.E. Peña leg. (AMNH); 1 \&, 6 imms, Parque Quinta Normal, 33.44071 S, 70.6826 W, in a house, May 1985, O. León leg. (MNHNCL); 1 q, Quilicura, 33.36667 S, 70.75 W, May 1979, L. Peña leg. (AMNH); 2 우, ditto, Oct. 1979 (AMNH); 1 ○, Parque Quinta Normal, 33.44056 S, $70.6825 \mathrm{~W}$, in house, behind a portrait, May 2002, O. León leg. (MNHNCL); 2 우, 2 imms, ditto, 33.45 S, 70.7 W, 1984, O. León leg. (MNHNCL); 1 ㅇ, ditto, 1985 (MNHNCL); 1 ㅇ, 1 imm., ditto (MNHNCL); 2 우, Renca, 33.38333 S, 70.7 W, 3 Oct 1983, Trarrazaval leg. (AMNH); 1 ㅇ, ditto, G. Mann leg. (AMNH); 2 + $ᄋ$, ditto, 33.45 S, $70.66667 \mathrm{~W},(\mathrm{AMNH}) ; 2$ 우, 2 imms, ditto, $33.36667 \mathrm{~S}, 70.75 \mathrm{~W}$ (AMNH); 1 ㅇ, ditto, (AMNH);

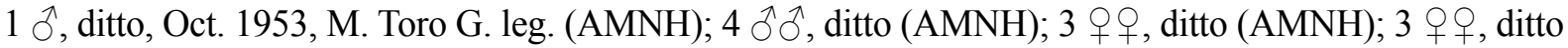
(AMNH); 3 ठึ, 3 우, 1 imm., ditto, 33.45 S, 70.7 W, 1 Oct. 2009, T.H. Kawamoto leg. (IBSP 161391). - V Región de Valparaíso: Marga Marga, 1 ô, 1 imm., Olmué, Olmué, 32.99103 S, 71.17318 W, in a house, 31 Jan. 2013, H. Wood and C. Griswold leg. (CAS 9053184; CAS 9053251); Valparaíso, 1 q,
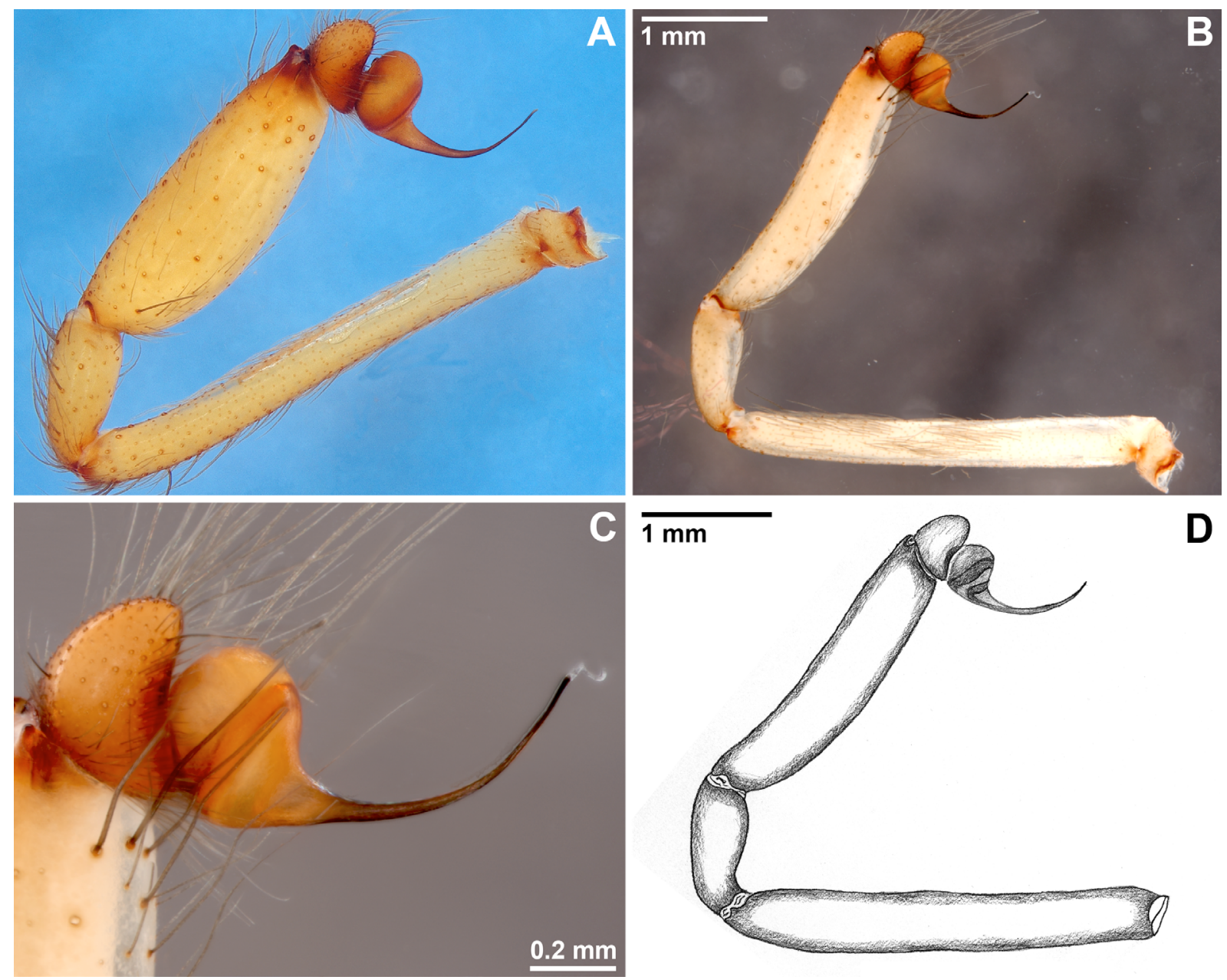

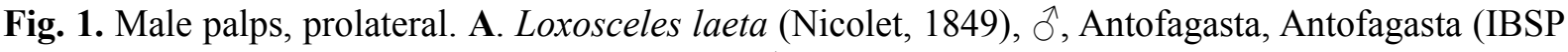
4663). B-D. Loxosceles surca Gertsch, 1967. B. §’, Chusmiza, Huara, Tamarugal, Tarapacá (AMNH). C. Same, bulb, prolateral. D. Same, palp, prolateral. Scale bars A-B $=1 \mathrm{~mm}$. 
Melipilla, Casablanca, 33.3 S, 71.38333 W, Jan.-Mar. 1955, E. Reed leg. (AMNH); 2 +,$~ 1$ imm., ditto, 33.31667 S, 71.41667 W, no date, G. Tobar leg. (MNRJ 632); 4 q, 2 imms, Quillota, Ocoa, Borda del Parque Nacional La Campana, 32.915 S, 71.09033 W, 355 m a.s.l., 23 Mar. 2013, A.D. Brescovit et al. leg. (IBSP 163704); 1 §̂, 1 q, 3 imms, Valparaíso, 32.88333 S, 71.26667 W, Jan. 1979, A. Tobar leg. (AMNH); 1 q, 1 imm., ditto, 33.06306 S, 71.63333 W, Feb. 1954, E. Reed leg. (AMNH); 1 q, Quintero, Valparaíso, Las Dunas, 32.93806 S, 71.55639 W, 11-12 May 1962, Donoso and Archer leg. (AMNH); 1 q, 1 imm., San Felipe, San Felipe, 32.706 S, 70.73733 W, 670 m a.s.l., 6 Apr. 2013, A.D. Brescovit and I.L.F. Magalhães leg. (IBSP 163718); 1 + , Valparaíso, Cascabelles, 31.99045 S, 71.50905 W, 14 Nov. 2013, A.D. Brescovit and I.L.F. Magalhaes leg. (IBSP 167088); 1 § , 1 q, Sector El Carretón, 32.11667 S, 71.26667 W, 26 Feb. 2006, F. Perez leg. (LEULS); 1 + , Viña del Mar, 33.03333 S, 71.53333 W, Jan. 1979, A. Tobar leg.

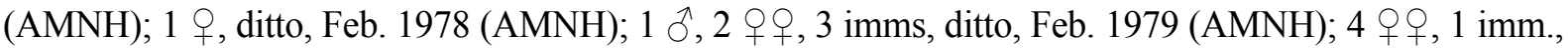

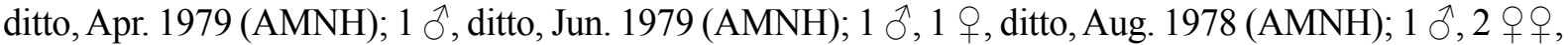
ditto, Oct. 1978 (AMNH); 1 ô, 1 q, 1 imm., ditto, Dec. 1978 (AMNH); 1 , ditto, 27 Jul. 1965 (IBSP 1946). - VII Región del Maule: Talca, 1 ㅇ, Talca, 35.43306 S, 71.66667 W, in a house, Sep.-Nov. 2003, C. Muñoz leg. (CAS 9053262). - VIII Región del Bío-Bío: Concepción, 1 q, Concepción, Campus Universidad de Concepción, 36.8275 S, 73.03889 W, 7 Dec. 1998, J. Artigas leg. (UCCC); 1 q, ditto, 36.83333 S, 73.05 W, 29 Dec. 1995 (UCCC); 1 9, ditto, 36.8275 S, 73.03333 W, 12 Jan. 1986, M. Marchant leg. (UCCC); 1 +, 3 imms, ditto, 20 Sep. 2004, J. Artigas leg. (UCCC); 1 + , ditto, 3 Oct. 2004 (UCCC); 1 q, ditto, 6 Oct. 2004 (UCCC); 1 \%, ditto, 36.83333 S, 73.05 W, 18 Feb. 2003 (UCCC); 1 đ, ditto, 25 Mar. 1985, S. Cekalovic leg. (AMNH). - IX Región de la Araucanía: Cautín, 1 đ̊, Temuco, Temuco, 38.75 S, $72.83333 \mathrm{~W}$, no date, no collector (MNHNCL). - X Región de los Lagos: Osorno, 1 + , Osorno, Purranque, 40.91667 S, 73.16667 W, Jan.-Mar. 1955, E. Reed leg. (AMNH). - XIV Región de los Ríos: Valdivia, 1 imm., Valdivia, Valdivia, 39.8 S, 73.23333 W, Apr. 1985, C. Arraigada leg. (MNHNCL). - XV Región

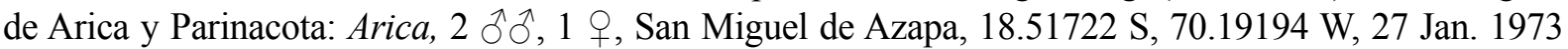
(AMNH); 1 , 1 imm., Lluta River Valley, 18.41139 S, 70.31972 W, GuicMann leg. (AMNH); 1 §, 2 우, Poconchile, 18.45083 S, 70.07017 W, 11 m a.s.1., 12 Jul. 2012, A.D. Brescovit, A.J. Santos and A. TaucareRíos leg. (IBSP 167063); 1 ð, 2 우, Valle de Azapa, 3 km S of San Miguel Azapa, 18.53417 S, 70.0 W, 24 Jan. 2014, A. Taucare-Ríos leg. (IBSP 167069); 1 क, ditto (IBSP 167068); 1 imm., ditto, $18.51667 \mathrm{~S}$, 70.18333 W, urban area, 27 May 1980, N. Hidrino leg. (UCCC); 1 Ô, ditto, 28 Dec. 1988, B. Roth leg. (CAS 9057745); 2 우우, ditto, 18.475 S, 70.31444 W, 27 Feb. 1986 (UCCC); 2 imms, ditto, 18.51667 S, 70.18333 W, 1980, J. Artigas leg. (UCCC); 1 imm., ditto, 21 May 1980, N. Hidrino leg. (UCCC); 1 imm., ditto, 26 Feb. 1980 (UCCC); 1 Oे, ditto (UCCC); 2 imms, ditto, 18.51722 S, 70.17611 W, 22 May 1980, Nelson leg. (UCCC); 1 imm., ditto, in a house, 27 May 1980, J. Artigas leg. (UCCC); 1 q, ditto, W, 1 Apr. 1980, Nelson leg. (UCCC); 1 imm., ditto, in a house, 1 Apr. 1980 (UCCC); 1 imm., ditto, 27 Feb. 1980, N. Hidrino leg. (UCCC); 1 + , Camarones, Taltape, Camarones Valley, 19.145 S, 70.17306 W, 29 Jan. 1973 (AMNH); Arica, 1 ㄱ, 2 우우, Arica, $3 \mathrm{~km}$ S of San Miguel de Azapa, 18.53417 S, 70.0 W, 24 Jan. 2014, A. Taucare-Ríos leg. (IBSP); 1 + , ditto, 24 Jan. 2015 (IBSP); 1 ô, 1 ㅇ, 12 imms, Valle de Azapa, 18.475 S, 70.31444 W, 800 m a.s.1., 29-31 Jan. 1992, L.E. Peña leg. (AMNH); Parinacota, 4 우, 1 imm., Putre, $100 \mathrm{~km}$ E of Zapahuira, 18.35333 S, 69.61833 W, 2003, A.A. Ojanguren Affilastro leg. (MACN 21408);

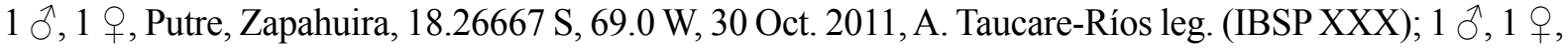
Ticnamar, 18.58167 S, 69.49444 W, 3200 m a.s.l., Mar. 1983, H. Niemeyer leg. (MNHNCL); Tarapacá, 1 ○ૈ, 2 우, , Arica, Valle del Río Lluta, 18.41056 S, 70.11889 W, 2 Feb. 1992, L.E. Peña leg. (AMNH). 1 ㅇ, no further data (MACN 21477); 1 , ditto (MNRJ 14601); 4 우, ditto (IBSP 34949); 1 , , ditto (MNRJ 2304).

\section{Description}

Male and female

Described by Gertsch (1967). 


\section{Distribution}

Widespread in Chile, in all Regions from Arica y Parinacota to Los Lagos, except Regions VI(O'Higgins), XI (Aisén) and XII (Magallanes) (Fig. 10). For records from other countries, see Gertsch (1967).

Loxosceles surca Gertsch, 1967

Figs 1B-D, 8A, 9B, 10

Loxosceles surca Gertsch, 1967: 157, pl. 8, figs 4, 6, 12 (female holotype from Peru, Lima, Surco, Rimac Valley, 11.8849 S, 76.4411 W, 2000 m a.s.1., 9 Oct. 1963, A. Herrer and P. Wygodzinsky leg., deposited in AMNH, not examined). — Taucare-Ríos 2011: 45, figs 1, 3. — World Spider Catalog 2017.

\section{Note}

Males and females were collected next to each other in at least three different localities.

\section{Diagnosis}

Loxosceles surca could be confused in Chile only with the sympatric L. laeta. It is distinguished from L. laeta by the anterior border of the carapace very hirsute, the male palp with tibia narrowed medially, with a dorsal concavity, and five times longer than the cymbium (Fig. 1B-C). The female internal genitalia are very similar to those of $L$. laeta, but can be distinguished by the almost absent transverse enlargement at the base of the tubular receptacles (Fig. 8A).

\section{Material examined}

CHILE. I Región de Tarapacá: Tamarugal, 1 q, 10 imms, Huara, Chusmiza, 19.68333 S, 69.18333 W, 3-250 m a.s.l., 10 Nov. 1992, L.E. Peña leg. (AMNH). - XV Región de Arica y Parinacota: Arica, 3 우, Arica, San Miguel de Azapa, 18.53533 S, 70.14983 W, 342 m a.s.l., 12 Jul. 2012, A.D. Brescovit, A.J. Santos and A. Taucare-Ríos leg. (IBSP 167059); 1 , , Playa Corazones, next to the Cuevas de Anzotas, 18.5482 S, 70.3307 W, 23 m a.s.1., 12 Jul. 2012, A.D. Brescovit, A.J. Santos and A. Taucare-Ríos leg. (IBSP 167061); Parinacota, 1 ภे, 3 q 우, Putre, Chapiquiña, 18.39472 S, 69.53694 W, 12 Nov. 1983 (AMNH); 2 우, $2 \mathrm{~km} \mathrm{~S}$ of Zapahuira, $18.33333 \mathrm{~S}, 69.56667 \mathrm{~W}, 3400 \mathrm{~m}$ a.s.l., 3 Feb. 1994, N.I. Platnick et al. leg. (AMNH); 1 o, 1 imm., 24 km S of Zapahuira, 18.41667 S, 69.51667 W, $3640 \mathrm{~m}$ a.s.l., 3 Feb. 1997, N.I. Platnick et al. leg. (AMNH); 1 đo, 1 imm., ditto, 3 Feb. 1998 (AMNH); 1 q, 6 km S of Zapahuira, 18.35 S, 69.56667 W, 3420 m a.s.l., 3 Feb. 1996, N.I. Platnick et al. leg. (AMNH); 1 \%, $9 \mathrm{~km} \mathrm{~S}$ of Zapahuira, 18.35 S, 69.53333 W, $3620 \mathrm{~m}$ a.s.l., 5 Feb. 1994 , N.I. Platnick et al. leg. (AMNH).

\section{Description}

Male (Zapahuira, Putre, Parinacota, Chile, AMNH)

Carapace yellowish, with the darker, violin-shaped median band typical of Loxosceles (Taucare-Ríos 2011: fig. 3). Endites and labium yellow. Sternum yellowish. Legs yellow with tibia, metatarsus and tarsus brown. Palp red brown, with distal articles reddish brown. Opisthosoma grey, with inconspicuous cardiac area. Total length 9.8. Caparace 3.8 long, 4.4 wide. Clypeus 0.55 high, with border hirsute. Eye diameters: ALE 0.2, PME 0.24, PLE 0.22. Sternum 2.3 long, 1.9 wide. Leg I: femur 6.0, patella 1.5, tibia 6.5, metatarsus 6.9, tarsus 2.2 , total 23.1. II: $6.8,1.6,7.1,7.7,2.0,25.2$. III: 6.2, 1.6, 5.6, 6.8, 1.7, 21.9 . IV: 6.9, 1.6, 7.0, 8.2, 2.0, 25.7. Tibiae sinuous, with ventral erect setae (Fig. 9B). Leg formula 4213. Palpal femur 5.2 long, 0.5 wide. Palpal tibia 2.8 long, 0.6 wide. Palp with cymbium as long the tegular base, and a long, slender and curved embolus (Fig. 1C).

\section{Female}

Described by Gertsch (1967). Clypeus as in male. Palp tarsus 1.5 long, narrow. Spermathecae with tubular receptacle straight, with sclerotized base (Fig. 8A). 


\section{Variation}

Male $(\mathrm{n}=3)$ : Total length $8.0-10.0$, carapace 4.1-4.4 long, femur I 5.6-6.8 long. Palpal tibia 2.8-3.0 long, $0.5-0.6$ wide. Female $(\mathrm{n}=8)$ : total length $10.0-12.4$, carapace $4.8-5.4$ long, femur I 5.2-5.6 long. Palpal tarsus 1.4-1.6 long.

\section{Distribution}

Known only from northern Chile, in Tarapacá and Arica y Parinacota Regions (Fig. 10) and from Peru: Santiago de Surco and Chosica (Gertsch 1967).

\section{Loxosceles coquimbo Gertsch, 1967}

Figs $2,8 \mathrm{~B}, 10$

Loxosceles coquimbo Gertsch, 1967: 158, pl. 8, fig. 5 (female holotype from Chile, IV Región de Coquimbo, Coquimbo, Elqui, Playa Blanca, 30.2218 S, 71.40602 W, deposited in AMNH, not examined).

\section{Note}

Males were not collected with females; the only male comes from a locality $83 \mathrm{~km}$ to the north of the nearest collected female. They are here associated based on the morphology of genital and copulatory organs, which are comparatively simple in the male and the female, and fitting the diagnosis of the laeta species-group, as well as on the leg formula (4213). Despite having visited the type locality, we could not obtain any topotypes.

\section{Diagnosis}

Loxosceles coquimbo differs from other Chilean species by the male palp with tegulum globose and embolus short and sinuous at tip (Fig. 2A-B). Females can be recognised by the inner and outer spermathecae with nearly the same size (Fig. 8B; Gertsch 1967: pl. 8, fig. 5).

\section{Material examined}

CHILE. IV Región de Coquimbo: Coquimbo, 2 q $q$, Coquimbo, Lomas de Peñuelas, 29.9333 S, 71.3 W, $8 \mathrm{~m}$ a.s.1., under rocks and under dead cactus, 6 Jan. 1985, N.I. Platnick and O. Francke leg. (AMNH); Elqui, 1 ภ, La Higuera, Los Choros, 29.2469 S, 71.4617 W, 6-9 Jul. 2007, J. Pizarro leg. (LEULS).
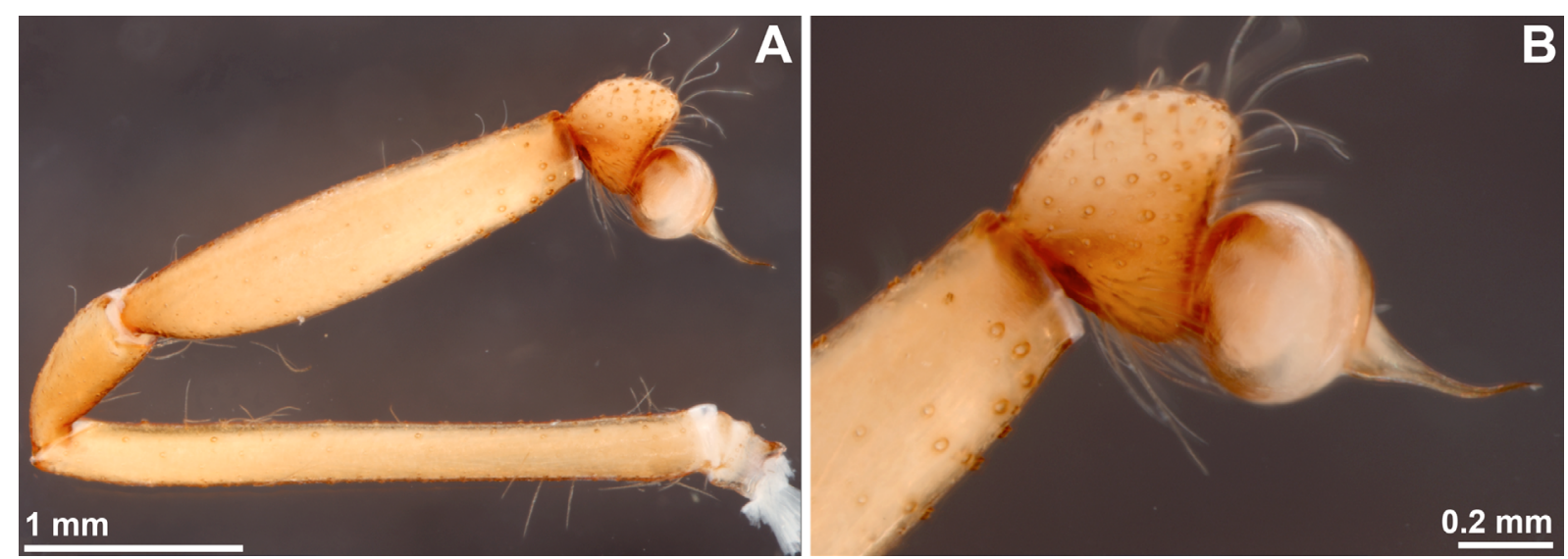

Fig. 2. Loxosceles coquimbo Gertsch, 1967, §̂, Los Choros, La Higuera, Elqui (LEULS). A. Palp, prolateral. B. Bulb, prolateral. 


\section{Description}

Male (Los Choros, Elqui, Chile, LEULS)

Specimen discoloured, but with carapace orange anteriorly and laterally, yellowish posteriorly. Chelicerae red brown. Endites, labium and sternum yellow. Legs yellowish. Palp yellow, except tibiae and cymbium orange. Opisthosoma uniformly grey. Total length 7.3. Caparace 3.3 long, 2.7 wide. Clypeus 0.35 high. Eye diameters: ALE 0.14, PME 0.16, PLE 0.16. Sternum 1.8 long, 1.4 wide. Leg I: femur 6.6, patella 1.1, tibia 7.5, metatarsus 7.5, tarsus 1.6, total 23.1. II: 7.5, 1.2, 8.6, 9.1, 1.8, 25.2. III: 6.2, 1.1, 6.1, 7.2, 1.3, 21.9. IV: $7.3,1.1,7.0,8.6,1.6,25.7$. Leg formula 4213. Palpal femur 3.1 long, 0.4 wide. Palpal tibia 2.1 long, 0.5 wide. Palp with a short cymbium, a round tegular base with half-length of cymbium, and a straight and short embolus with a truncated tip (Fig. 2).

\section{Female}

Described by Gertsch (1967). Palpal tarsus narrow.

\section{Variation}

Female $(\mathrm{n}=2)$ : total length $6.3-7.2$, carapace $2.4-3.3$ long, femur I 3.6-3.8 long. Palpal tarsus 1.2-1.3 long.

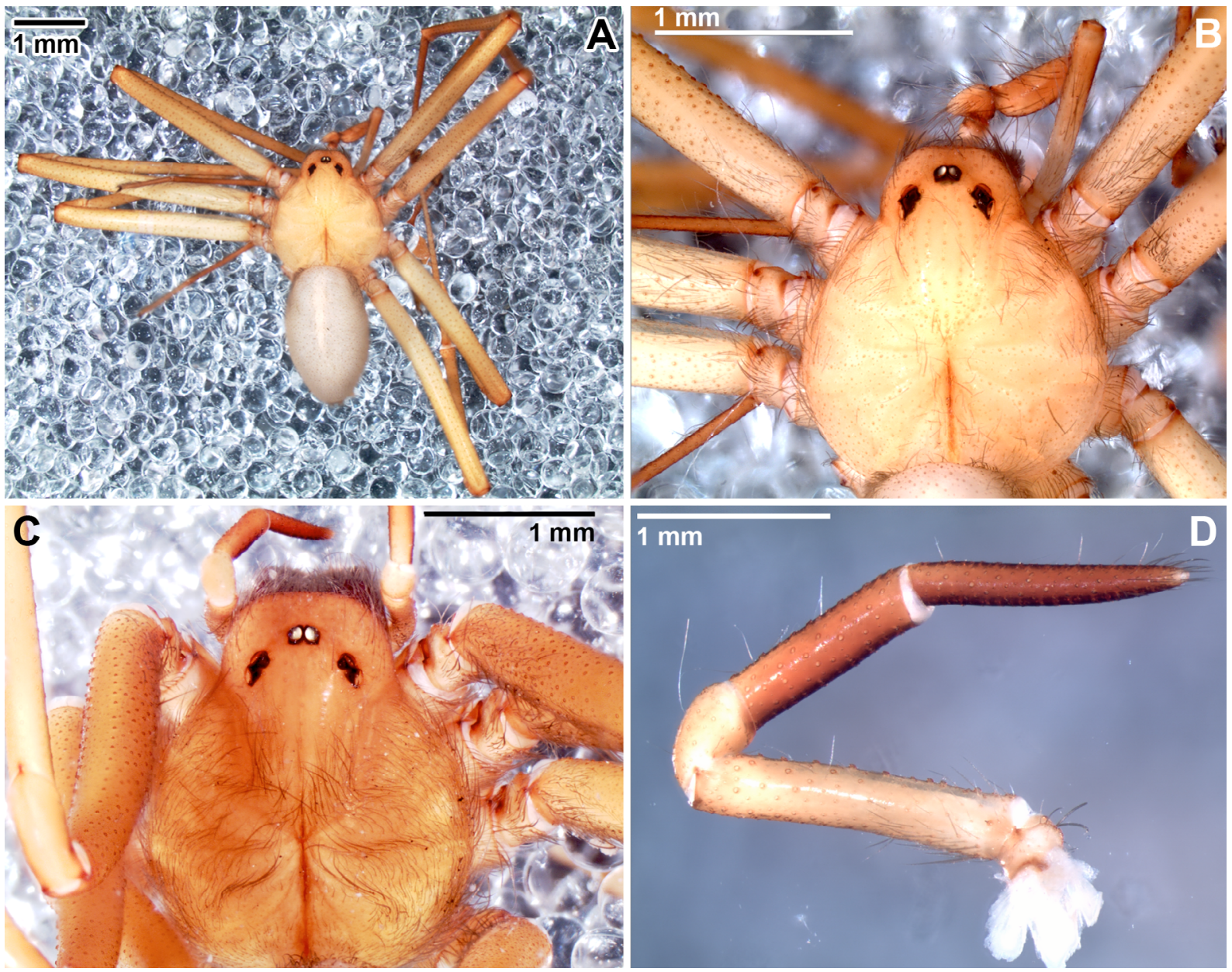

Fig. 3. Loxosceles diaguita sp. nov. A-B. ${ }^{\Uparrow}$, holotype (MNHNCL 7946). A. Habitus, dorsal. B. Carapace, dorsal. C-D. o, paratype (MACN-Ar 30185). C. Carapace, dorsal. D. Left palp, prolateral. 


\section{Distribution}

Known from the Coquimbo Region in Chile only (Fig. 10).

\section{Loxosceles diaguita sp. nov.}

urn:lsid:zoobank.org:act:D3A0FA32-AC7E-4C34-9830-9A1C599653B4

Figs 3-6, 8D, 10

\section{Diagnosis}

Males are easily distinguished from other Neotropical Loxosceles by the shape of the palpal bulb, with a large embolus sided by a keel with several longitudinal grooves (Figs 3C-D, 6A-C). Females are distinguished by the robust, S-shaped stalks of the outer spermathecae and by the reduced inner spermathecae with long, thin stalks (Fig. 8D).

\section{Etymology}

The Diaguitas are a Native American tribe whose original territory covered northwestern Argentina and northern Chile, including portions of the Antofagasta Region, the type locality of the species. The name is to be treated as a noun in apposition.
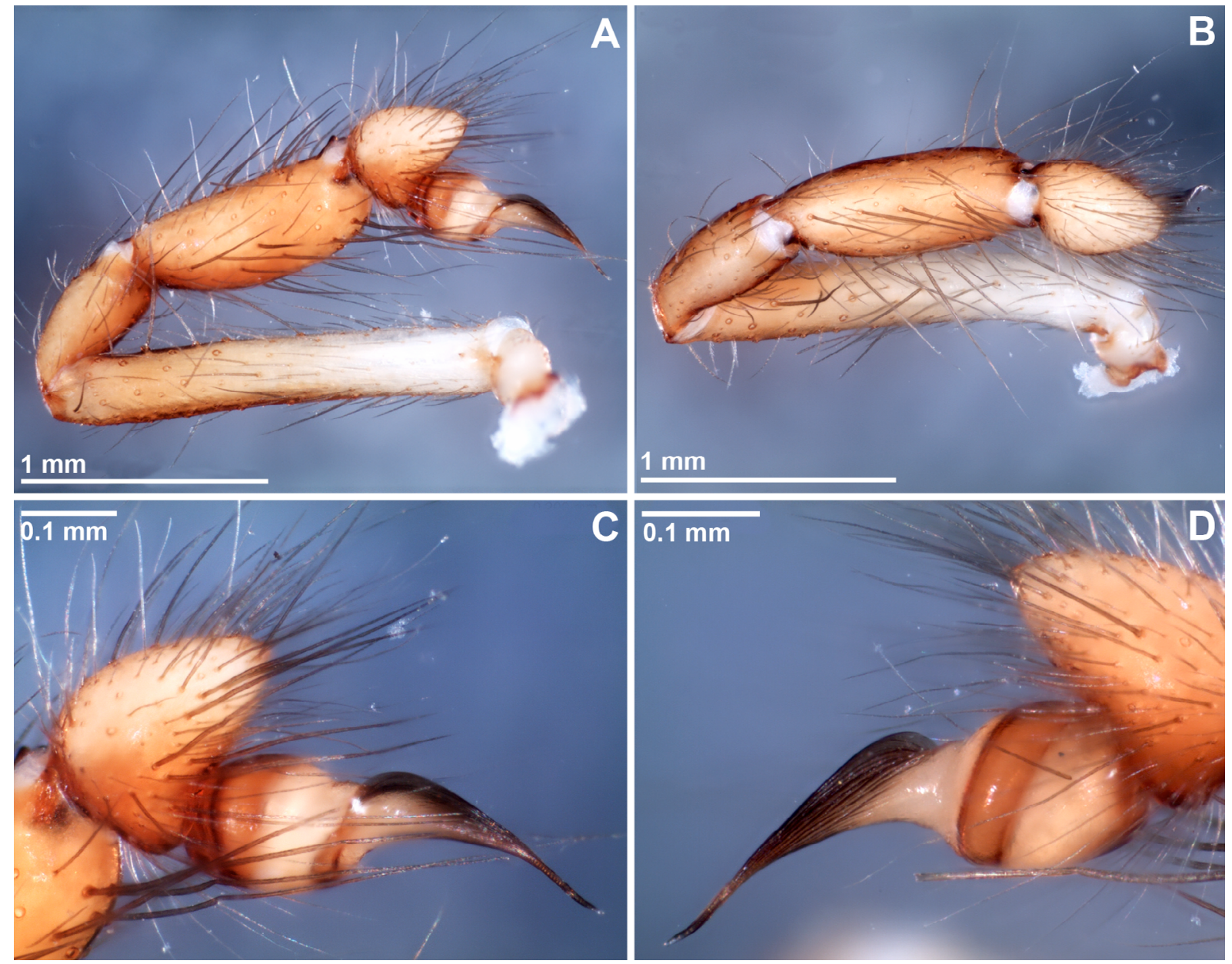

Fig. 4. Loxosceles diaguita sp. nov., ठ̂, holotype (MNHNCL 7946). A-D. Left palp. A. Prolateral. B. Dorsal. C. Bulb, prolateral. D. Bulb, retrolateral. 


\section{Type material}

\section{Holotype}

CHILE: ㄱ, II Región de Antofagasta, Antofagasta, Taltal, $8.1 \mathrm{~km} \mathrm{NNW}$ of Paposo, $24.93947 \mathrm{~S}$, $70.49265 \mathrm{~W}, 114 \mathrm{~m}$ a.s.1., (MJR-loc-45), desert in bloom, matorrales, manual collecting, 27-29 Oct. 2011, M.J. Ramírez, A. Ojanguren and J. Pizarro leg. (MNHNCL 7946).

\section{Paratypes}

CHILE: 2 우, same data as for holotype (MACN-Ar 30185, MNHNCL 7947); 1 §, 1 q, Taltal, $6 \mathrm{~km} \mathrm{E} \mathrm{of}$ Paposo, 25.25 S, 70.25 W, 480 m a.s.1., 12 Oct. 1992, N. Platnick, R. Catley and P. Goloboff leg. (AMNH); 2 우, Taltal, 4 km E of Paposo, 25.3 S, 70.2 W, 20-50 m a.s.1., 11 Oct. 1992, N. Platnick, R. Catley and P. Goloboff leg. (AMNH); 1 P, Paposo, campamento base, 25.00767 S, 70.44678 W, 601 m a.s.1., 26-29 Oct. 2015, R. Botero-Trujillo, J.R. Pizarro-Araya, J.E. Barriga-Tuñón and F.A. Kong leg. (MACN-Ar 37212).

\section{Note}

\section{Males and females}

Males and females have been matched because they have been collected in the same locality in two different expeditions.

\section{Description}

\section{Male (holotype, MNHNCL 7946)}

Carapace, labium, sternum and legs light brown; carapace lacking the dark-coloured violin-shaped median band typical of Loxosceles (Fig. 3A-B). Endites light brown, distally reddish brown. Palp light brown, with distal articles reddish brown. Opisthosoma light gray, with whitish cardiac area. Total length 7.31. Caparace 3.33 long, 3.06 wide. Clypeus 0.42 high. Eye diameters: ALE 0.17, PME 0.17, PLE 0.18. Sternum 1.76 long, 1.5 wide. Leg I: femur 6.38, patella 1.24, tibia 6.52, metatarsus 6.52, tarsus 1.47 , total 22.13. II: 7.32, 1.3, 8.65, 7.98, 1.53, 26.78. III: 5.85, 1.22, 5.85, 5.85, 1.3, 20.07. IV: 6.38, 1.25, $6.38,7.05,1.5,22.56$. Leg formula 2413. Palpal femur 1.83 long, 0.28 wide. Palpal tibia 0.97 long, 0.4 wide. Palp with a short cymbium, a round and short tegular base, and a large and curved embolus with a sculptured keel running from its base to the tip (Figs 4, 6).

\section{Female (paratype, MACN-Ar 30185)}

Colour as in male, carapace hirsute (Fig. 3C). Total length 9.71. Caparace 4.26 long, 3.72 wide. Clypeus 0.57 high. Eye diameters: ALE 0.18, PME 0.18, PLE 0.2. Sternum 2.36 long, 1.93 wide. Leg I: femur 6.12, patella 1.43, tibia 6.92, metatarsus 5.99, tarsus 1.4, total 21.86. II: 6.92, 1.52, 7.71, 6.78, 1.4, 24.33 . III: $5.85,1.44,5.45,5.72,1.34,19.8$. IV: $6.52,1.47,6.52,5.05,1.47,21.03$. Leg formula 2143. Palp tarsus 1.54 long, narrow (Fig. 3D). Spermathecae with a wide, sclerotized base. Inner spermathecae
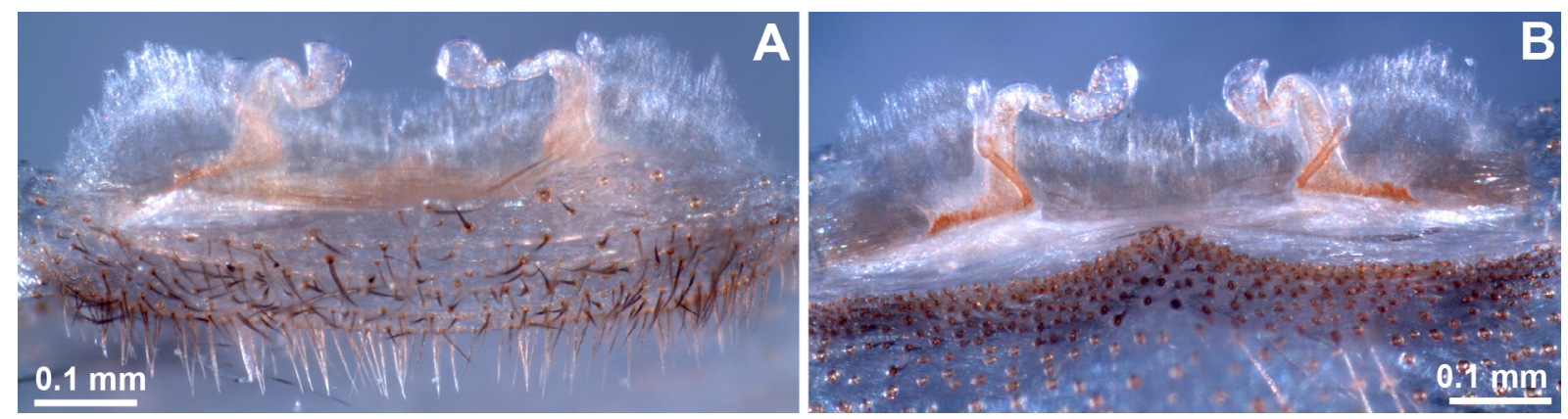

Fig. 5. Loxosceles diaguita sp. nov., + , paratype (MACN-Ar 30185). A-B. Spermathecae. A. Dorsal. B. Ventral. 
with long and thin stalks, which are laterally directed so that the outer spermathecae receptacles are placed medially relative to the inner spermathecae receptacles. Outer spermathecae larger than the inner spermathecae, with a robust, S-shaped stalk (Figs 5, 8D).

\section{Variation}

Male $(\mathrm{n}=2)$ : Total length 7.3-7.6, carapace 3.3-3.8 long, femur I 6.2-6.38 long. Palpal tibia 0.9-1.1 long, 0.4-0.5 wide. Female $(\mathrm{n}=4)$ : total length 9.7-10.8, carapace 4.0-4.4 long, femur I 5.8-6.4 long. Palpal tarsus 1.2-1.54 long.

\section{Distribution}

Known from three nearby localities near Paposo, Antofagasta, and northern Chile (Fig. 10).

\section{Loxosceles pallalla sp. nov.}

urn:1sid:zoobank.org:act:97475BEE-48BC-4AAB-A32C-A4E165B26973

Figs $7,8 \mathrm{C}, 10$

\section{Diagnosis}

Loxosceles pallalla sp. nov. can be distinguished from all Loxosceles species by the small tegular base and extremely long embolus with a flattened keel in the male palp (Fig. 7). Females can be recognised
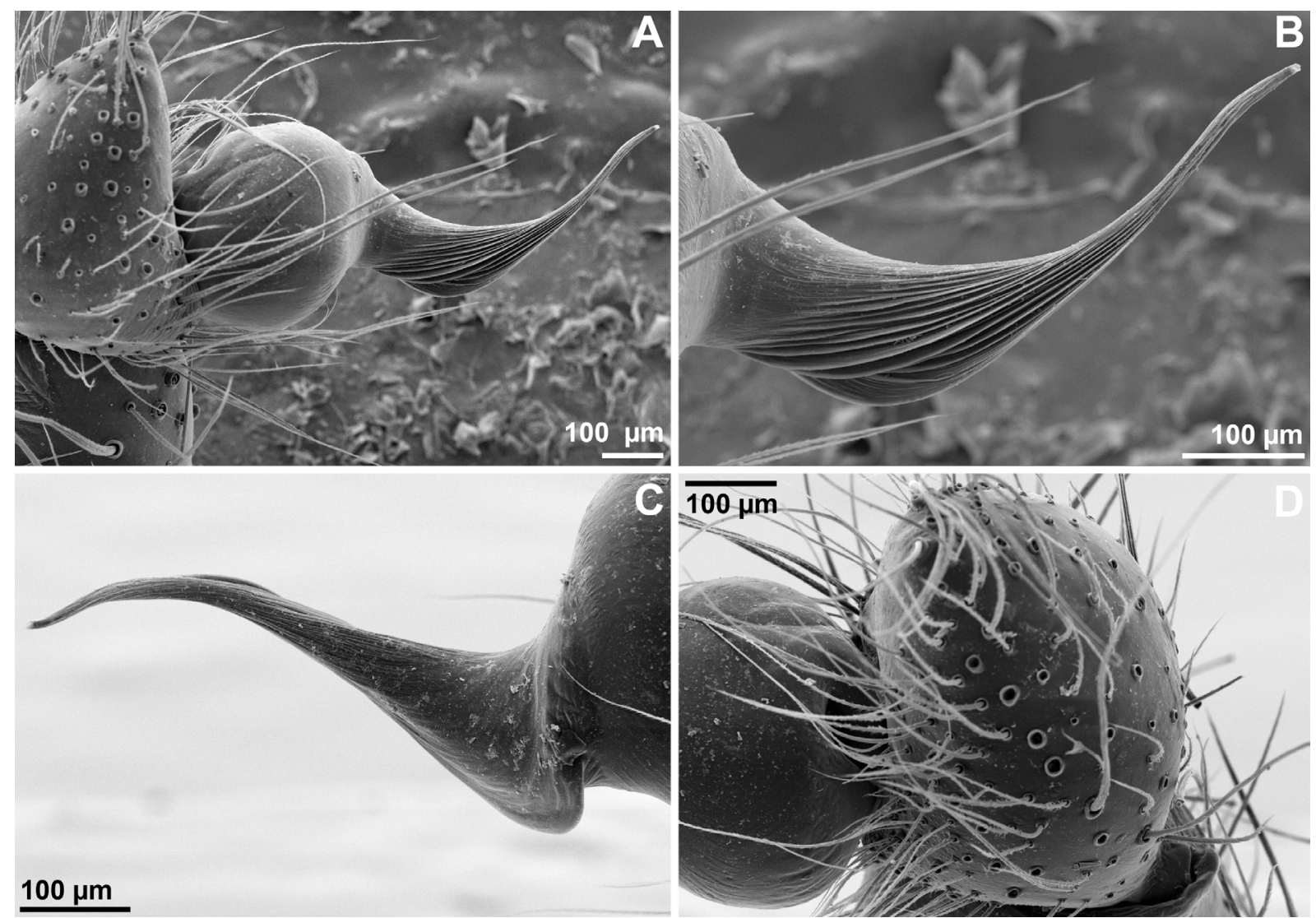

Fig. 6. Loxosceles diaguita sp. nov., $\widehat{\jmath}$, Taltal, $4 \mathrm{~km}$ E of Paposo, Antofagasta (AMNH), SEM. A-D. Left palp. A. Prolateral. B. Detail of embolus, prolateral. C. Bulb, retrolateral. D. Cymbium, retrolateral. 
by the spermathecae with two receptacles originating from the same short and curved stalk, and the inner spermathecae larger than the outer one (Fig. 8C).

\section{Etymology}

The specific epithet means "flat" in the Aymara language, and refers to the flattened embolus of this species.

\section{Type material}

\section{Holotype}

CHILE: đ̊ , IV Región de Coquimbo: Elqui, La Herradura, 30.48333 S, 71.6 W, 80 m a.s.1., 3 Oct. 1992, N. Platnick, P. Goloboff and K. Catley leg. (AMNH).

\section{Paratype}

CHILE: , Elqui, $6 \mathrm{~km} \mathrm{~S}$ of Cruz Grande, 29.31667 S, $71.31667 \mathrm{~W}, 5 \mathrm{~m}$ a.s.1., in the sand by the beach, 11 Nov. 1993, N.I. Platnick, K. Catley, M. Ramírez and R.T. Allen leg. (AMNH).
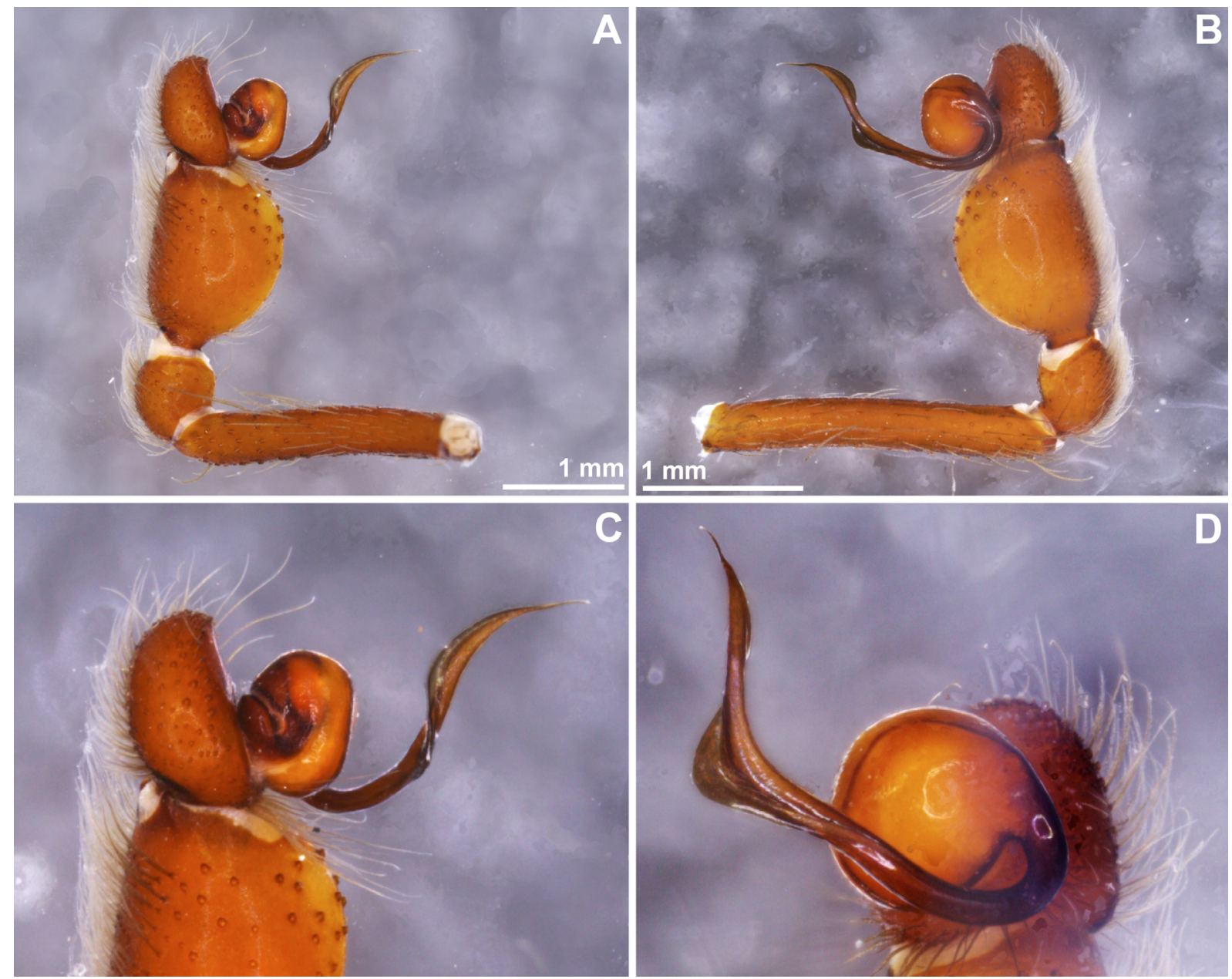

Fig. 7. Loxosceles pallalla sp. nov., đ̂, holotype, La Herradura, Elqui, IV Región de Coquimbo, Chile (AMNH). A. Palp, prolateral. B. Palp, retrolateral. C. Bulb, prolateral. D. Bulb, subventral. 


\section{Note}

Males and females were matched because they have the same coloration and have leg formula 2413 . Also, both males and females have a deviant genital morphology, not fitting in any of Gertch's speciesgroups. Although this species may be sympatric with Loxosceles coquimbo, the latter are easily separated by the leg formula 4213 .

\section{Description}

\section{Male (holotype, AMNH)}

Carapace orange with cephalic area red brown, lacking the dark-coloured, violin-shaped median band. Chelicerae red brown. Labium, endites and legs orange. Sternum yellow. Palpus red brown. Opisthosoma uniformly grey, with spinnerets orange. Total length 7.2. Caparace 3.4 long, 2.8 wide. Clypeus 0.4 high, with long setae in the border. Eye diameters: ALE 0.16, PME 0.16, PLE 0.18. Sternum 1.7 long, 1.4 wide. Leg I: femur 5.9, patella 1.1, tibia 6.2, metatarsus 6.3, tarsus 1.6, total 21.1. II: 7.1, 1.1, 8.2, 8.3, 1.6, 26.3. III: 5.3, 1.0, 5.0, 5.7, 1.1, 18.1. IV: 6.6, 1.1, 6.2, 7.5, 1.5, 22.9. Leg formula 2431. Palpal femur 2.2 long, 0.4 wide. Palpal tibia 1.1 long, 0.8 wide. Palp with short cymbium, half the length of tibia, tibia short, enlarged (Fig. 7A-B); tegular base as large as the cymbium, embolus extremely long, coiled near base and flattened in all extension, except the slender tip (Fig. 7C-D).

Female (paratype, AMNH)

Colour as in male. Total length 8.6. Caparace 3.3 long, 3.0 wide. Clypeus 0.4 high, as in male. Eye diameters: ALE 0.16, PME 0.16, PLE 0.16. Sternum 1.8 long, 1.5 wide. Leg I: femur 5.4, patella 1.2, tibia 5.7, metatarsus 5.3, tarsus 1.3, total 18.9. II: 6.0, 1.2, 6.2, 6.1, 1.4, 20.9. III: 5.2, 1.1, 4.6, 4.9, 1.2, 17.0. IV: $5.9,1.2,5.6,6.3,1.4,20.4$. Leg formula 2413. Palp tarsus 1.3 long, narrow. Spermathecae small, positioned farther apart, without a single sclerotised base (Fig. 8C).

\section{Distribution}

Known only from the Coquimbo Region, Chile (Fig. 10).

Loxosceles vallenar sp.nov. urn:1sid:zoobank.org:act:C3AAF160-148A-483A-8976-7391C4754F29

Figs $8 \mathrm{E}, 10$

\section{Diagnosis}

Females of Loxosceles vallenar sp. nov. can be distinguished from other Chilean species by the spermathecae with large base and slender, curved receptacles (Fig. 8E).

\section{Etymology}

The specific name is a noun in apposition taken from the type locality.

\section{Type material}

\section{Holotype}

CHILE: +, III Región de Atacama: Huasco, Vallenar, $3 \mathrm{~km} \mathrm{~S}$ of Vallenar, 28.56667 S, $70.75 \mathrm{~W}$, $460 \mathrm{~m}$ a.s.1., in scrubby mountain-side, under rocks, 7 Jan. 1985, N. Platnick and O. Francke leg. (AMNH).

\section{Description}

Male

Unknown. 
Female (holotype, AMNH)

Carapace anteriorly and laterally orange, posteriorly yellow, lacking the dark-coloured violin-shaped median band. Chelicerae red brown. Endites and labium orange. Sternum, legs and coxae, femur and patella of palps yellow. Palpal tibia and tarsus red brown. Opisthosoma uniformly grey. Total length 6.0. Carapace 2.2 long, 1.6 wide. Clypeus 0.25 high. Eye diameters: ALE 0.1, PME 0.12, PLE 0.1. Sternum 1.2 long, 0.9 wide. Leg I: femur 2.1, patella 0.6 , tibia 2 , metatarsus 1.8 , tarsus 0.7 , total 7.2. II: $2.2,0.6$, 2.0, 1.8, 0.7, 7.3. III: 1.9, 0.6, 1.5, 1.6, 0.5, 6.1. IV: 2.3, 0.7, 2.2, 2.1, 0.7, 8.0. Leg formula 4213. Palp tarsus 0.7 long, enlarged. Spermathecae with oval receptacle (Fig. 8E).
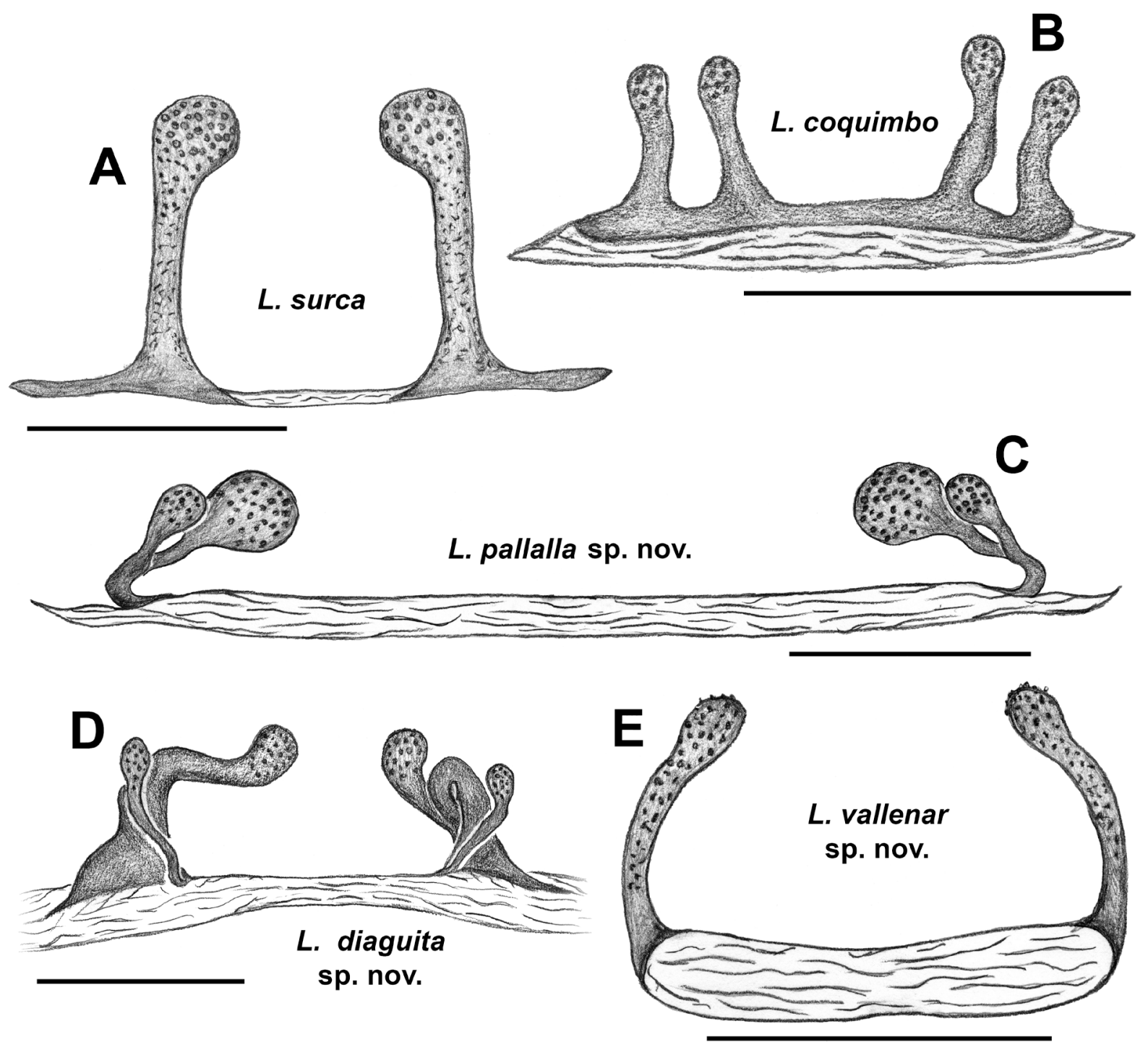

Fig. 8. Spermathecae, dorsal view (except D, ventral), cleared. A. Loxosceles surca Gertsch, 1967, +, Zapahuira, Parinacota, Tarapacá (AMNH). B. Loxosceles coquimbo Gertsch, 1967, + , Lomas de Peñuelas, Coquimbo (AMNH). C. Loxosceles pallalla sp. nov.,,$+6 \mathrm{~km} \mathrm{~S}$ of Cruz Grande, Elqui (AMNH). D. Loxosceles diaguita sp. nov.,, , paratype (MACN-Ar 30185). E. Loxosceles vallenar sp. nov.,, , holotype $(\mathrm{AMNH})$. Scale bars $=0.5 \mathrm{~mm}$. 


\section{Distribution}

Known only from the type locality in the Atacama Region, Chile (Fig. 10).

\section{Discussion}

\section{Relationships}

Gertsch (1967) proposed the gaucho, spadicea, amazonica and laeta groups in the genus Loxosceles. These morphological groups are still accepted and have so far resisted scrutiny from molecular (Binford et al. 2008) and morphological (Magalhaes et al. 2017) phylogenetic analyses. The Chilean Loxosceles fauna was known only by two species (World Spider Catalog 2017) and is here expanded with records of $L$. surca and three new species, two of which cannot be unambiguously assigned to any of Gertch's groups.

Loxosceles laeta and L. surca are undoubtedly included in the laeta group by the original definition of Gertsch (1967), mainly based on the long palpal tibia and elongated embolus (Fig. 1A-B, D). These characters are easily observed in the species of this group distributed in Ecuador, Peru, northern Chile
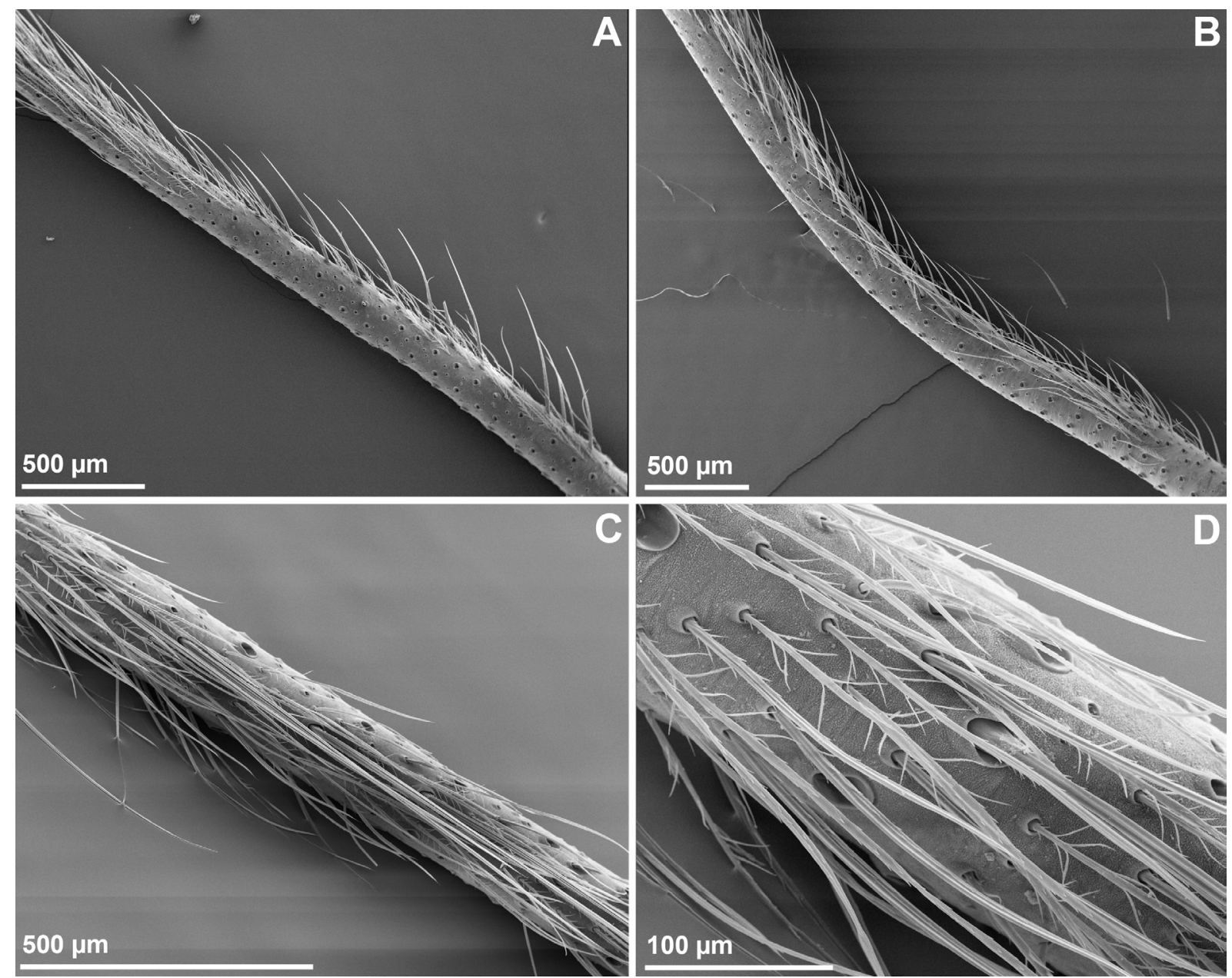

Fig. 9. Scanning electron microscopy, ổ metatarsus I. A. Loxosceles laeta (Nicolet, 1849), ठ̊,, Antofagasta, Antofagasta (IBSP 4663). B. Loxosceles surca Gertsch, 1967, Õ, Parinacota, $24 \mathrm{~km} \mathrm{~S}$ of Zapahuira (AMNH). C-D. Loxosceles diaguita sp. nov., Ô, Taltal, 4 km E of Paposo, Antofagasta (AMNH). 
and Argentina and southern Brazil. Loxosceles coquimbo was described based on a female and was included in the laeta group by Gertsch (1967) mainly based on the leg formula, the thickened pedipalp and, strangely, on the pair of tubular receptacles on each side of the transverse atrium (Fig. 8B). The genitalia of the females of the laeta-group are more variable than those of the males. The inclusion of Loxosceles coquimbo in the group seems reasonable with the discovery of the male, which has a long palpal tibia (Fig. 2A-B).

Among the new species here described, Loxosceles vallenar sp. nov. could also be a member of the laeta-group, pending confirmation upon discovery of the male. The female has the leg formula 4213 as well as tubular receptacles on each side of the elongated and transverse atrium, both are characteristics of this group (Fig. 8E).

Loxosceles diaguita sp. nov. shares two characters with the spadicea-group: the shape of the spermathecae (with inner spermathecae placed externally to the larger outer spermathecae; compare with Magalhaes et al. 2017: fig. 63C) and a keel in the embolus (Magalhaes et al. 2017: fig. 63B). However, it does not have the typical, apomorphic morphology of the male bulb of this group (rather short and spherical), nor the much reduced inner spermathecae (see Gertsch 1967). This suggests that L. diaguita sp. nov. might represent the sister group of all other species in the spadicea-group. Curiously, it occurs west of the Andes, while all other species of the group occur east of this mountain range.

Finally, Loxosceles pallalla sp. nov. is the most peculiar of the herein described species, with a long and flattened embolus originating from the retrolateral side of the bulb in the male palp (Fig. 7) and with female genitalia having two receptacles arising from a single stalk (Fig. 8C). Based on the male palp with a short cymbium and a relatively short and dilated tibia (Fig. 7A-B), and following Gertsch's (1967) classification, this species should be included in the rufescens or gaucho-groups. However, the
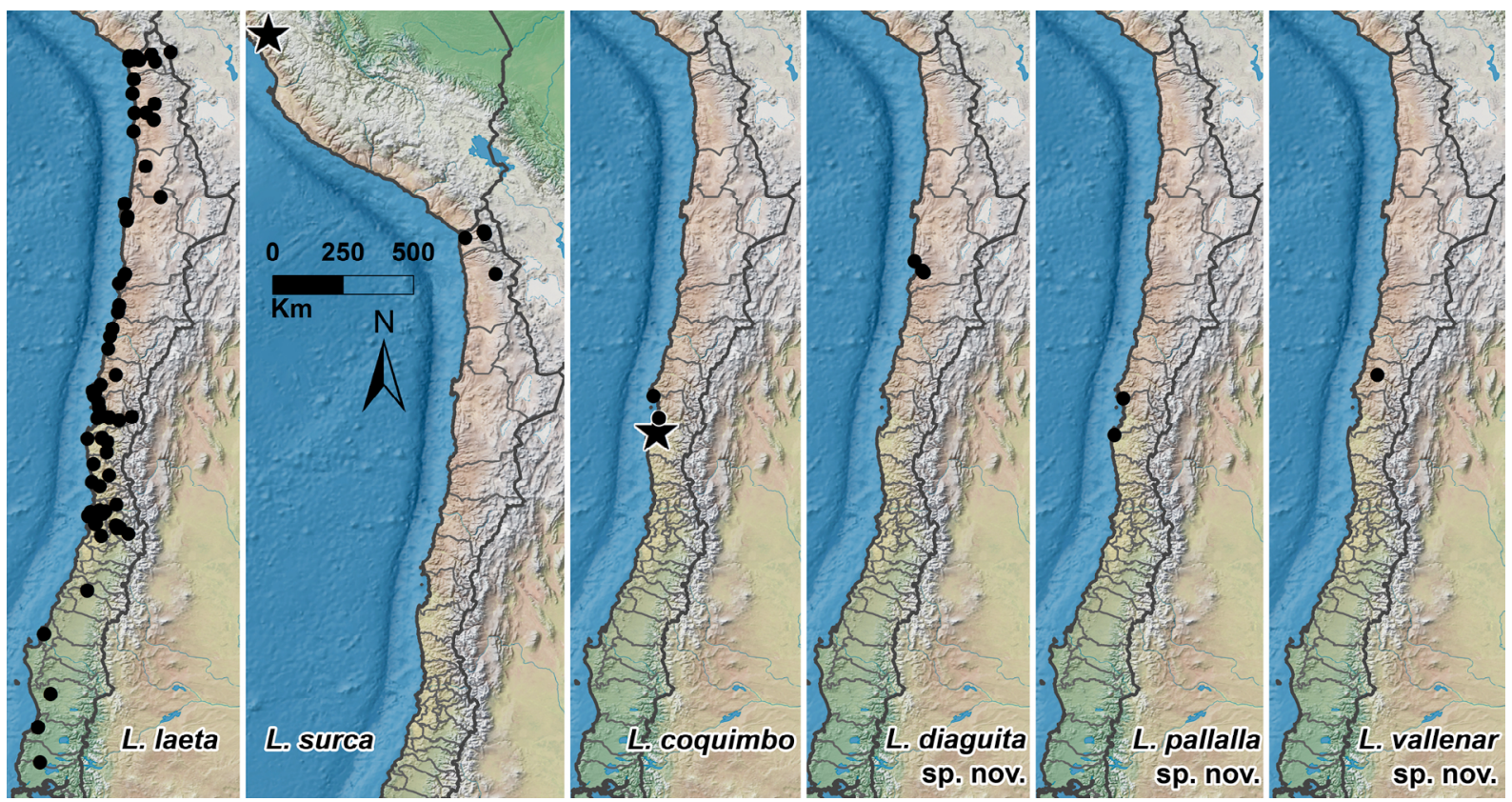

Fig. 10. Geographic distribution records of species of Loxosceles in Chile. Records of L. laeta (Nicolet, 1849) are based on the material examined in this study and on Gertsch (1967). Stars under L. surca Gertsch, 1967 and L. coquimbo Gertsch, 1967 are records from Gertsch (1967). 
female genital morphology is quite unmodified, and does not resemble the highly apomorphic female morphology of any of these groups (with sclerotized plates in the gaucho-group, and with several apical lobes in the the rufescens-group, see Fukushima et al. 2017). Additionally, the keel in the male palp could be a shared feature between this species and the spadicea-group. It would be very interesting to include L. pallalla sp. nov. in a phylogenetic analysis to test its position in a quantitative way. At any rate, its discovery demonstrates that the Chilean fauna may still hold an undiscovered Loxosceles diversity.

\section{Diversity}

In this paper we recognize six species, three of them endemic to the Chilean transitional coastal desert. The transitional coastal desert of Chile $\left(25-32^{\circ} \mathrm{S}\right)$ represents the northern limit of the biodiversity hotspot recognized for Central Chile (Gaston 2000), and is a major area of endemism for restrictedly distribute arthropods (Pizarro-Araya \& Flores 2004; Cepeda-Pizarro et al. 2005; Agusto et al. 2006; Ojanguren-Affilastro et al. 2007). The discovery of these new species confirms the importance of this area for biological conservation (Cepeda-Pizarro et al. 2005). On the other hand, Loxosceles surca and L. vallenar sp. nov. are restricted to arid inland environments of northern and central Chile, respectively, while $L$. laeta is widely distributed and would be the only synanthropic species in the country (TaucareRíos et al. 2013).

\section{Acknowledgements}

Thanks to Beatriz Mauricio, the operator of the Centro de Microscopia Eletronnica at the Instituto Butantan, and to the curators of the collections cited above for lending important material. This study was financially supported by grants to ADB (CNPq 301776/2004-0 and FAPESP 2011/50689-0), a CONICET doctoral fellowship and grants to ILFM (CONICET: PIP 2012-0943 and FONCyT: PICT 2011-1007), to AJS (FAPEMIG: PPM-00651-15; CNPq: 407288/2013-9, 306222/2015-9; and Instituto Nacional de Ciência e Tecnologia dos Hymenoptera Parasitóides da Região Sudeste Brasileira (http:// www.hympar.ufscar.br/)) and to ATR (CONICYT grant 21130014 doctoral fellowship).

\section{References}

Agusto P., Mattoni C.I., Pizarro-Araya J., Cepeda-Pizarro J. \& López-Cortés F. 2006. Comunidades de escorpiones (Arachnida: Scorpiones) del desierto costero transicional de Chile. Revista Chilena de Historia Natural 79: 407-421. https://doi.org/10.4067/S0716-078X2006000400001

Binford G.J., Callahan M.S., Bodner M.R., Rynerson M.R., Núñez P.B., Ellison C.E. \& Duncan R.P. 2008. Phylogenetic relationships of Loxosceles and Sicarius spiders are consistent with Western Gondwanan vicariance. Molecular Phylogenetics and Evolution 49: 538-553.

https://doi.org/10.1016/j.ympev.2008.08.003

Cepeda-Pizarro J., Pizarro-Araya J. \& Vásquez H. 2005. Composición y abundancia de artrópodos del Parque Nacional Llanos de Challe: impactos del ENOS de 1997 y efectos del hábitat pedológico. Revista Chilena de Historia Natural 78: 635-650. https://doi.org/10.4067/S0716-078X2005000400004

Fischer M.L., Grosskopf C.B., Bazílio S. \& Ricetti J. 2011. Araneofauna sinantrópica associada com a família Sicariidae no município de União da Vitória, Paraná, Brasil. Sitientibus, Ciências Biológicas 11: $48-56$.

Fukushima C.S., Andrade R.M.G. \& Bertani R. 2017. Two new Brazilian species of Loxosceles Heinecken \& Lowe, 1832 with remarks on amazonica and rufescens groups (Araneae, Sicariidae). ZooKeys 667: 67-94. https://doi.org/10.3897/zookeys.667.11369

Gaston K.J. 2000. Global patterns in biodiversity. Nature 405: 220-227. https://doi.org/10.1038/35012228 
Gertsch W.J. 1967. The spider genus Loxosceles in South America (Araneae, Scytodidae). Bulletin of the American Museum of Natural History 136: 117-174.

Magalhaes I.L.F., Brescovit A.D. \& Santos A.J. 2017. Phylogeny of Sicariidae spiders (Araneae: Haplogynae), with a monograph on Neotropical Sicarius. Zoological Journal of the Linnean Society 179: 767-864.

Nicolet H. 1849. Arácnidos. In: Gay, C. (ed.) Historia fisica y política de Chile. Zoología, vol. 3. Paris, pp. 319-543. https://doi.org/10.5962/bhl.title.61752

Ojanguren-Affilastro A.A., Agusto P., Pizarro-Araya J. \& Mattoni C.I. 2007. Two new scorpion species of genus Brachistosternus (Scorpiones: Bothriuridae) from northern Chile. Zootaxa 1623: 55-68. https://doi.org/10.5281/zenodo.179242

Pizarro-Araya J. \& Flores G.E. 2004. Two new species of Gyriosomus Guérin-Méneville from Chilean coastal desert (Coleoptera: Tenebrionidae: Nycteliini). Journal of the New York Entomological Society 112: 121-126. https://doi.org/10.1664/0028-7199(2004)112[0121:TNSOGG]2.0.CO;2

Sánchez-Ruiz A. \& Brescovit A.D. 2013. The genus Loxosceles Heineken \& Lowe (Araneae: Sicariidae) in Cuba and Hispaniola, West Indies. Zootaxa 3731: 212-222. https://doi.org/10.11646/zootaxa.3731.2.2

Schenone H. 2004. A propósito del loxoscelismo en Chile. Revista Médica de Chile 132: 121-122. https://doi.org/10.4067/S0034-98872004000100018

Schenone H., Rojas A., Reyes H., Villarroel F. \& Suarez G. 1970. Prevalence of Loxosceles laeta in houses of central Chile. The American Journal of Tropical Medicine and Hygiene 19: 564-567.

Schenone H., Saavedra T., Rojas A. \& Villarroel F. 1989. Loxoscelismo en Chile: estudios epidemiológicos, clínicos y experimentales. Revista do Instituto de Medicina Tropical de São Paulo 31 (6): 403-415. https://doi.org/10.1590/S0036-46651989000600007

Senff-Ribeiro A., Silva P.H., Chaim O.M., Gremski L.H., Paludo K.S., Silveira R.B., Gremski W., Mangili O.C. \& Veiga S.S. 2008. Biotechnological applications of brown spider (Loxosceles genus) venom toxins. Biotechnology Advances 26: 210-218. https://doi.org/10.1016/j.biotechadv.2007.12.003

Silva A.M., Bernarde P.S. \& Abreu L.C. 2015. Accidents with poisonous animals in Brazil by age and sex. Journal of Human Growth and Development 25 (1): 54-62. https://doi.org/10.7322/jhgd.96768

Silva P.H., Silveira R.B., Appel M.H., Mangili O.C., Gremskia W. \& Veiga S.S. 2004. Brown spiders and loxoscelism. Toxicon 44: 693-709. https://doi.org/10.1016/j.toxicon.2004.07.012

Simon E. 1900. Liste des arachnides recueillis par M. Ch. E. Porter en 1898-1899 et descriptions d'espèces nouvelles. Revista Chilena de Historia Natural 4: 49-55.

Taucare-Ríos A. 2011. Loxosceles surca (Gertsch, 1967) (Araneae: Sicariidae) en el norte de Chile. Boletín de Biodiversidad de Chile 5: 45-49.

Taucare-Ríos A., Brescovit A. \& Canals M. 2013. Synanthropic spiders (Arachnida: Araneae) from Chile. Revista Ibérica de Aracnología 23: 49-56.

Vetter R.S. 2008. Spiders of the genus Loxosceles (Araneae, Sicariidae): a review of biological, medical and psychological aspects regarding envenomations. Journal of Arachnology 36: 150-163.

https://doi.org/10.1636/RSt08-06.1

World Spiders Catalog. 2017. World Spiders Catalog, v. 18.5. Natural History Museum Bern. Available from http://wsc.nmbe.ch [accessed 14 Apr. 2017]. 
Manuscript received: 23 February 2017

Manuscript accepted: 16 June 2017

Published on: 27 December 2017

Topic editor: Rudy Jocqué

Desk editor: Pepe Fernández

Printed versions of all papers are also deposited in the libraries of the institutes that are members of the EJT consortium: Muséum national d'Histoire naturelle, Paris, France; Botanic Garden Meise, Belgium; Royal Museum for Central Africa, Tervuren, Belgium; Natural History Museum, London, United Kingdom; Royal Belgian Institute of Natural Sciences, Brussels, Belgium; Natural History Museum of Denmark, Copenhagen, Denmark; Naturalis Biodiversity Center, Leiden, the Netherlands; Museo Nacional de Ciencias Naturales-CSIC, Madrid, Spain; Real Jardín Botánico de Madrid CSIC, Spain. 